\title{
Broadband Full-Wave BIE Impedance Characterization of 3-D Interconnects
}

\author{
Martijn Huynen, Daniël De Zutter, Dries Vande Ginste \\ Electromagnetics Group/IDLab, Department of Information Technology \\ Ghent University/imec, Ghent, Belgium \\ martijn.huynen@ugent.be,daniel.dezutter@ugent.be,dries.vandeginste@ugent.be
}

\begin{abstract}
In this paper, a full-wave boundary integral equation formulation is proposed that accurately characterizes 3-D interconnects. By extending a recently presented framework for the resistance and inductance calculation that employs a 3-D differential surface admittance operator, the total impedance is computed. The inclusion of capacitive effects is validated and demonstrated over a broad frequency range.

Index Terms-3-D surface admittance, boundary integral equation (BIE), interconnect modeling.
\end{abstract}

\section{INTRODUCTION}

The proliferation of electronic devices in a wide variety of applications has driven electronic circuits and systems to increasing complexity and diminishing size. These evolutions bring about a set of challenges for the design engineer, especially in terms of signal and power integrity. To assess and tackle these problems, electromagnetic solvers are widely utilized. However, the models at the core of such solvers are often based on approximations. Improved, full-wave models are thus increasingly important to obtain reliable simulation results.

Electromagnetic full-wave solvers come in all shapes and sizes and can be classified in various ways. A common distinction is made based on the discretization approach of the method, viz., volume or surface discretization. The former category houses, among others, the well-known finite element method (FEM). By meshing the entire 3-D volume, this method provides a versatile tool for a wide range of applications. However, volume discretization requires a very large number of (small) mesh elements to properly represent phenomena such as the skin and proximity effect in good conductors, leading to computationally intractable systems.

By only meshing the boundary of the structure, surface discretization schemes, such as boundary integral equation (BIE) methods, result in considerably smaller systems at the cost of a dense system matrix. Nevertheless, taking into account good conductors remains a challenging feat. A popular class of approximate techniques employs surface impedances to model conductors [1]. Other methods actually directly solve the full-wave inner problem in the conducting medium [2] but suffer from numerical inaccuracies and computational complexity due to the behavior of the Green's function [3]. An approach that solves this troublesome problem utilizes a differential surface admittance operator [4], constructed using the eigenmodes of the conductor's shape.

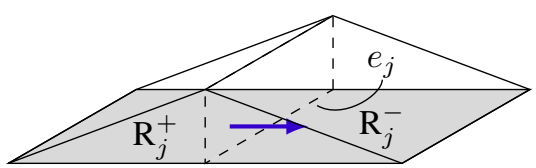

Fig. 1. Rooftop function of edge $e_{j}$ with its support $S_{j}=R_{j}^{+} \cup R_{j}^{-}$.

In this paper, we extend our novel framework [5] employing a 3-D differential surface admittance for characterizing interconnect structures to include capacitive effects. This is achieved by constructing a set of matrix equations analogous to the approach followed in [6] for the electric field integral equation (EFIE). This way, for the first time, a complete broadband surface equivalent representation of good conductors is obtained.

\section{Formulation OF THE METHOD}

Consider $N$ disjunct volumes $\mathcal{V}_{i}$, each filled with a (different) homogeneous, nonmagnetic material defined by its wavenumber $k_{i}$, embedded in a homogeneous, nonmagnetic background medium with wavenumber $k_{0}$. Applying the equivalence principle to each $\mathcal{V}_{i}$, we can replace the inner material by that of the background medium by introducing an unknown surface current density $\mathbf{j}_{s}$ on the boundary surface $\mathcal{S}_{i}$ which ensures that the fields outside $\mathcal{V}_{i}$, i.e., $\left(\mathbf{e}_{0}, \mathbf{h}_{0}\right)$, remain unchanged. This approach gets rid of the material discontinuities and results in a problem that is generally more suited to be solved with a BIE method as the Green's function of the restored background medium $G_{0}\left(\mathbf{r}, \mathbf{r}^{\prime}\right)$ is easier to construct/evaluate. In particular, we use the EFIE:

$$
\mathbf{e}_{0}=-j \omega \mathbf{a}-\nabla \phi,
$$

with a the magnetic vector potential and $\phi$ the electric scalar potential ( $e^{j \omega t}$ dependence).

Sticking to the BIE approach, we mesh all the boundary surfaces $\mathcal{S}_{i}$ that support the current densities. These surface meshes can be triangular with the corresponding RWG basis functions but as we focus on cuboids in the examples, we demonstrate the approach for rectangular meshes with the associated rooftops functions $\mathbf{b}_{j}$ for every edge $e_{j}$ as depicted in Fig. 1. Every rooftop is defined on two adjacent rectangles $R_{j}^{+}$and $R_{j}^{-}$, which together form the support $S_{j}$ of $\mathbf{b}_{j}$.

Expanding $\mathbf{e}_{0}$ and $\mathbf{j}_{s}$ into these rooftop functions, collecting the expansion coefficients in the vectors $\mathbf{E}$ and $\mathbf{I}$, respectively, 
and testing (1) with the same basis functions, leads to the matrix equation (for more details, see [5]):

$$
\overline{\bar{G}} \mathbf{E}=-j \omega \overline{\bar{L}} \mathbf{I}-\overline{\bar{T}} \mathbf{V},
$$

with the following definitions

$$
\begin{aligned}
(\overline{\bar{G}})_{i j} & =\int_{S_{i} \cap S_{j}} \mathbf{b}_{i} \cdot \mathbf{b}_{j} \mathrm{~d} S \\
(\overline{\bar{L}})_{i j} & =\mu_{0} \int_{S_{i}} \int_{S_{j}} G_{0}\left(\mathbf{r}, \mathbf{r}^{\prime}\right) \mathbf{b}_{i} \cdot \mathbf{b}_{j} \mathrm{~d} S \mathrm{~d} S^{\prime} \\
(\mathbf{V})_{i} & =\int_{R_{i}} \frac{\phi}{A_{i}} \mathrm{~d} S
\end{aligned}
$$

with $R_{i}$ being a rectangle in the mesh with area $A_{i}$. Each element in $\mathbf{V}$ represents the average potential on a single rectangle. The incidence matrix $\overline{\bar{T}}$ links the edges and patches of the mesh as follows:

$$
(\overline{\bar{T}})_{i j}= \begin{cases}1, & \text { if } R_{j} \text { is } R_{i}^{+} \text {of } \mathbf{b}_{i} \\ -1, & \text { if } R_{j} \text { is } R_{i}^{-} \text {of } \mathbf{b}_{i} \\ 0, & \text { otherwise. }\end{cases}
$$

Note that the Gramian matrix $\overline{\bar{G}}$ is block diagonal with every block the Gramiam matrix of $\mathcal{V}_{i}$. Matrix $\overline{\bar{L}}$, on the other hand, is dense due to the global nature of the Green's function.

At this point, we introduce the discretized differential surface admittance operator [7] to obtain a relation between $\mathbf{E}$ and I, viz., $\overline{\bar{G}} \mathbf{I}=\overline{\bar{Y}} \mathbf{E}$, and as such eliminate $\mathbf{E}$ from (2):

$$
\left(\overline{\bar{G}} \overline{\bar{Y}}^{-1} \overline{\bar{G}}+j \omega \overline{\bar{L}}\right) \mathbf{I}-\overline{\bar{T}} \mathbf{V}=0 .
$$

An additional connection between $\mathbf{I}$ and $\mathbf{V}$ is now constructed by introducing the expression for $\phi$, leading to $\mathbf{V}=$ $\overline{\bar{K}} \mathbf{Q}$ where $(\mathbf{Q})_{i}$ is the charge per rectangle $R_{i}$ and

$$
(\overline{\bar{K}})_{i j}=\frac{1}{\epsilon_{0}} \int_{R_{i}} \int_{R_{j}} \frac{G_{0}\left(\mathbf{r}, \mathbf{r}^{\prime}\right)}{A_{i} A_{j}} \mathrm{~d} S \mathrm{~d} S^{\prime} .
$$

To eliminate the newly introduced $\mathbf{Q}$, we invoke charge conservation on every rectangle. In other words, by discretizing $\nabla \cdot \mathbf{j}_{s}+j \omega \rho_{s}=0$ on every $R_{i}$, we get

$$
\overline{\bar{T}}^{T} \mathbf{I}+j \omega \mathbf{Q}=\mathbf{S},
$$

where $\mathbf{S}$ represents external current sources. Combining (7) and (9) results in a composite matrix equation:

$$
\left[\begin{array}{cc}
\overline{\bar{G}} \overline{\bar{Y}}^{-1} \overline{\bar{G}}+j \omega \overline{\bar{L}} & -\overline{\bar{T}} \\
\overline{\bar{T}}^{T} & j \omega \overline{\bar{K}}^{-1}
\end{array}\right]\left[\begin{array}{l}
\mathbf{I} \\
\mathbf{V}
\end{array}\right]=\left[\begin{array}{l}
\mathbf{0} \\
\mathbf{S}
\end{array}\right] .
$$

Solving this equation, either directly or iteratively, results in the currents on the edges of the mesh and the voltages on the rectangles. It can be utilized to determine, amongst other properties, the impedance response of the structure, as will be demonstrated in Section III.

So far we have assumed that our structure consists of $N$ disjunct volumes (all cuboids in the presented examples). In order to simulate relevant structures, however, the various

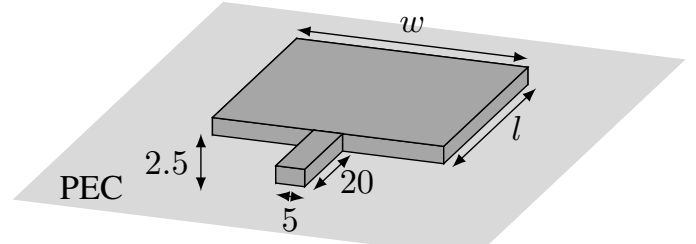

Fig. 2. Copper plate $\left(\sigma=5.8 \cdot 10^{7} \mathrm{~S} / \mathrm{m}\right)$ with a feed stub above a perfect electric conducting (PEC) half-space. The thickness of the conductor is $1 \mu \mathrm{m}$. All other dimensions are given in $\mu \mathrm{m}$ on the diagram.

TABLE I

CAPACitAnCE IN fF OF THE STRUCTURE IN Fig. 2

\begin{tabular}{ccc|ccc}
$1[\mu \mathrm{m}]$ & $\mathrm{w}[\mu \mathrm{m}]$ & $\alpha=l / w$ & $C_{\mathrm{pp}}$ & Yuan/Trick [8] & Eq. $(10)$ \\
\hline 50 & 50 & 1 & 9.208 & 12.035 & 12.647 \\
111.80 & 22.36 & 5 & 9.208 & 12.742 & 13.524 \\
22.36 & 111.80 & 0.2 & 9.208 & 12.742 & 13.462
\end{tabular}

cuboids should be combined into more complex shapes. We effectuate this connection by equating the voltage on adjacent rectangles of two cuboids and by introducing an additional unknown current between those two patches. In the presented matrix formalism, this boils down to the addition of an extra row and column per rectangle pair with two nonzero entries each. The column contains a 1 and -1 to include the extra current in the charge conservation equation on each rectangle while the additional row ensures the equality of both voltages.

\section{NUMERICAL EXAMPLES}

\section{A. Validation example}

Consider the geometry depicted in Fig. 2. A copper plate $\left(\sigma=5.8 \cdot 10^{7} \mathrm{~S} / \mathrm{m}\right)$ of dimension $l \times w$ with a thickness of $1 \mu \mathrm{m}$ is fed by a stub of $20 \mu \mathrm{m} \times 5 \mu \mathrm{m}$ and suspended $2.5 \mu \mathrm{m}$ above a perfect electric conducting ground plane. Initially we choose $l=w=50 \mu \mathrm{m}$ and use the simple parallel plate formula $\left(C_{\mathrm{pp}}=\epsilon A / d\right)$ for both cuboids to estimate the total capacitance of this structure (see Table I). However, this ignores the fringing fields and will as such underestimate the true capacitance. Various analytical expressions to estimate this effect can be found for 2-D and 3-D capacitors. We will employ the simple expressions for 2-D conductors with a finite thickness as found in [8] and apply them along two dimensions for an improved estimation. All results are collected in Table I. It is apparent that the parallel plate formula severely underestimates the total capacitance. Employing the analytical formulas results in a $30 \%$ increase while our fullwave solution computes a capacitance that is $37 \%$ larger. The additional increase found with the integral equation solution is due to the corners and the connection of both blocks.

According to the parallel plate formula, the capacitance only depends on the area of the plate and not on its shape. However, the analytical formulas contradict this and suggest that fringing effects do depend on the exact shape. Therefore, we investigate configurations with the same, fixed area for various aspect ratios $\alpha=l / w$. The difference between the calculated and nominal capacitance of $9.208 \mathrm{fF}$ is characterized by the fringing factor $\gamma$ such that $C=\gamma C_{\mathrm{pp}}$. For some 


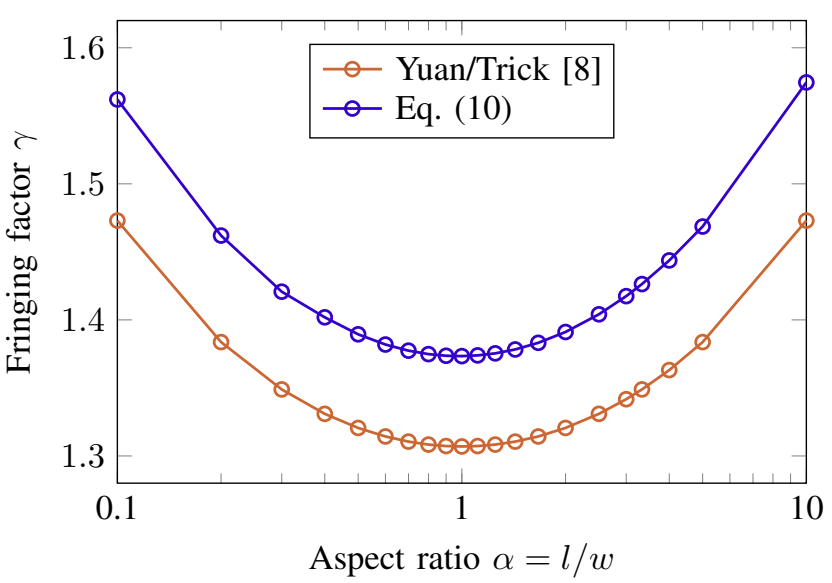

Fig. 3. Fringing factor $\phi$ for various aspect ratios $\alpha=l / w$.

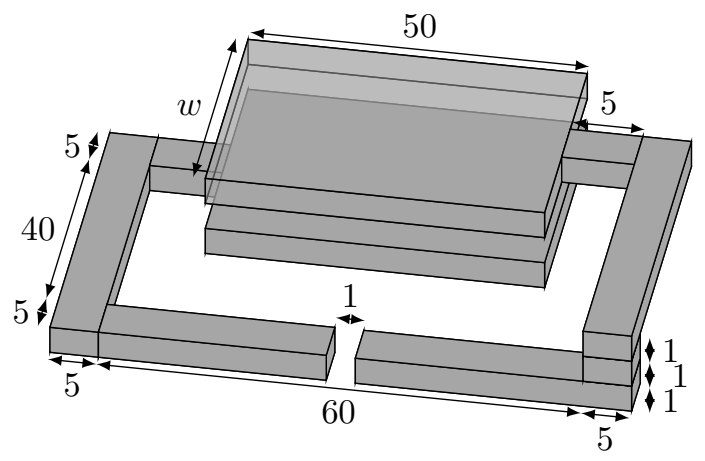

Fig. 4. Two copper plates of variable width $w$ with a feeding structure. All dimensions shown are in $\mu \mathrm{m}$. The impedance is measured over the $1 \mu \mathrm{m}$ gap.

specific values of $\alpha$ the capacitances are included in Table I. From Fig. 2 we see that $\gamma$ is minimum for a square plate and increases the more the plate is elongated in either direction. Note as well that the capacitance for reciprocal aspect ratios is slightly different according to the simulation results. Two such values are included in Table I and show that for longer plates the capacitance is marginally larger than for wider plates. This phenomenon can be attributed to the feed stub, which is aligned along the length of the plate, and a such introduces a small asymmetry.

\section{B. Application example}

The second example, depicted in Fig. 4, represents a copper parallel plate capacitor with variable width $w$ and a $1 \mu \mathrm{m}$ separation between the plates, fed via two traces that form a rectangular loop. All dimensions are annotated on the figure and are given in $\mu \mathrm{m}$. The total impedance of this structure is measured over the $1 \mu \mathrm{m}$ gap for a broad frequency range. In Fig. 5 the absolute value of this impedance is shown for various values of $w$ (including results from CST MWS shown with markers) and for a reference planar loop. This reference loop has the same shape and dimensions, i.e., $60 \mu \mathrm{m} \times 40 \mu \mathrm{m}$, as the structure in Fig. 4 but lacks the parallel plates (for a similar structure, see [5]). For low frequencies, we see that the structure behaves as a capacitance which increases for larger widths $w$. The response of the planar loop, on the

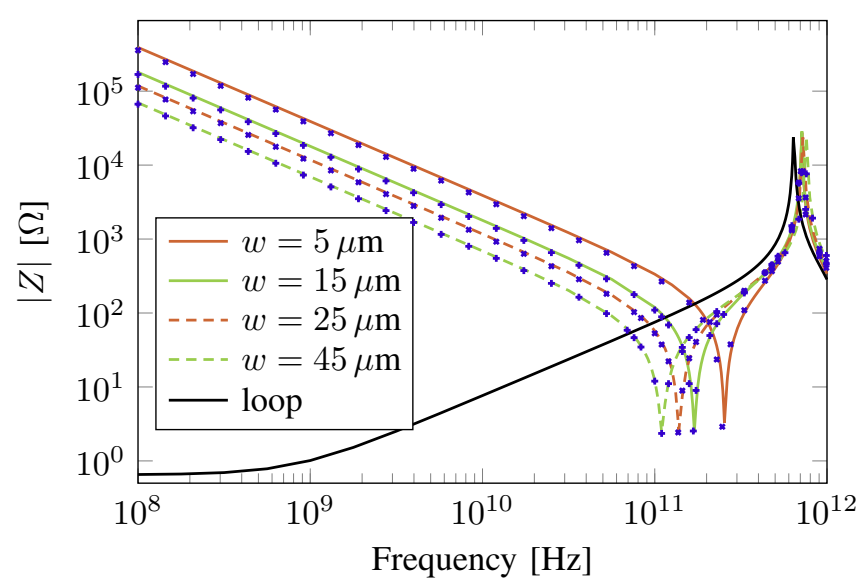

Fig. 5. Absolute value of the impedance of the structure in Fig. 4 for various values of $w$ with CST MWS results (blue markers) and the impedance of a reference loop (black).

other hand, indicates inductive behavior with a non-negligible resistive component that levels out the response for the lowest frequencies. Around $150 \mathrm{GHz}$, the structure undergoes a series resonance, whose precise frequency shifts with $w$ as the capacitance changes. For even higher frequencies (around $700 \mathrm{GHz}$ ), the structure endures a second (parallel) resonance. This phenomenon is caused by the capacitance of the gap where the impedance is measured. The planar loop exhibits the same resonance albeit at a lower frequency of $630 \mathrm{GHz}$ due the absence of the parallel plate capacitance.

\section{Conclusions}

A full-wave BIE method employing a differential surface admittance operator to extract the total impedance of 3-D interconnects was put forward. The formulation circumvents the cumbersome evaluation of the Green's function in good conductors and thus leads to accurate broadband results as demonstrated in the presented examples.

\section{REFERENCES}

[1] T. B. A. Senior and J. L. Volakis, Approximate boundary conditions in electromagnetics. IET, 1995.

[2] U. R. Patel, S. Sharma, S. Yang, S. V. Hum, and P. Triverio, "Fullwave electromagnetic characterization of 3D interconnects using a surface integral formulation," in 26th Conference on Electrical Performance of Electronic Packaging and Systems, San Jose, CA, 2017.

[3] J. Peeters, I. Bogaert, and D. De Zutter, "Calculation of MoM interaction integrals in highly conductive media," IEEE Trans. Antennas Propag., vol. 60, no. 2, pp. 930-940, Feb 2012.

[4] D. De Zutter and L. Knockaert, "Skin effect modeling based on a differential surface admittance operator," IEEE Trans. Microw. Theory Techn., vol. 53, no. 8, pp. 2526-2538, Aug 2005.

[5] M. Huynen, D. De Zutter, and D. Vande Ginste, "Rigorous full-wave resistance and inductance computation of 3-D interconnects," IEEE Microw. Wireless Compon. Lett., vol. 28, no. 6, pp. 455-457, June 2018.

[6] Z. G. Qian and W. C. Chew, "Fast full-wave surface integral equation solver for multiscale structure modeling," IEEE Trans. Antennas Propag., vol. 57, no. 11, pp. 3594-3601, Nov 2009.

[7] M. Huynen, M. Gossye, D. De Zutter, and D. Vande Ginste, "A 3-D differential surface admittance operator for lossy dipole antenna analysis," IEEE Antennas Wireless Propag. Lett., vol. 16, pp. 1052-1055, 2017.

[8] C. P. Yuan and T. N. Trick, "A Simple Formula for the Estimation of the Capacitance of Two-Dimensional Interconnects in VLSI Circuits," IEEE Electron Device Lett., vol. 3, no. 12, pp. 391-393, 1982. 